\title{
Reflex inhibition and reflex elicitation by acoustic stimuli differing in abruptness of onset and peak intensity
}

\author{
JAMES R. ISON \\ University of Rochester, Rochester, New York 14627
}

\begin{abstract}
The rat's acoustic startle reaction to a loud tone (S2) was inhibited by white-noise preliminary stimuli (S1) varying in rise/decay time (5 and $100 \mathrm{msec}$ ) and intensity $(70,80$, and $90 \mathrm{~dB})$. The amount of inhibition was linearly related to the intensity of S1 but was unaffected by its rise/ decay time. In contrast, the response directly elicited by S1 was affected by both variables, being greater with greater intensity and with the more abrupt onset. These data reveal that reflex elicitation and reflex inhibition are the products of different neural mechanisms.
\end{abstract}

This experiment questioned whether differential rise time of acoustic stimuli, a variable which has major effects on startle reflex elicitation, is important in determining the ability of a stimulus to inhibit reflexes elicited by a subsequent event. The theoretical concern behind the experiment was whether reflex inhibition and reflex elicitation are the products of the same or of different mechanisms, a concern which can be pursued in some measure by exploring the extent to which the two phenomena enter into similar empirical relationships.

The mammalian response to an intense sound burst is a rapid contraction of the flexor musculature, a reaction which may range from an involvement of the entire body to only a facial grimace and eyeblink, depending on the conditions of elicitation (Landis \& Hunt, 1939). The effectiveness of the sound in eliciting the acoustic startle reflex is partially determined by the abruptness of its onset. Thresholds for the whole-body flinch in the rat (Fleshler, 1965) and for the eyeblink in the human (Berg, 1973; cited in Graham, 1975) are lower for stimuli with faster rise times, and the temporal period over which the acoustic input is reflected in response strength is very short, approximating $12 \mathrm{msec}$ in the rat, $30 \mathrm{msec}$ in the human. An acoustic event which only gradually increases in strength is not a successful elicitor of the startle reflex.

The startle reflex in both rats and humans is inhibited if an extraneous initial stimulus, S1, is presented at certain lead times before the eliciting stimulus, S2 (Hoffman \& Searle, 1965; Ison \& Hammond, 1971; Krauter, Leonard, \& Ison, 1973).

\footnotetext{
This research was supported by a research grant from the National Institute of Neurological and Communicative Disorders and Stroke, NS-12443. Reprints may be obtained from Professor James R. Ison, Department of Psychology, Psychology Building, University of Rochester, Rochester, New York 14627.
}

Inhibition may be detected at intervals as long as $1 \mathrm{sec}$, but the time of maximum inhibition is on the order of 5 or $10 \times 10^{-2} \mathrm{sec}$. The initial stimulus can be effective if it is at the detection threshold (Reiter \& Ison, 1977); it may be auditory (Hoffman \& Searle, 1965), visual (Ison \& Hammond, 1971), or tactile (Pinckney, 1976); and it may be an offset as well as an onset of a stimulus (Stitt, Hoffman, \& Marsh, 1973). Thus it is clear that the occurrence of inhibition does not require that the initial stimulus actually elicit an acoustic startle reaction.

It has been suggested, however, that S1 may elicit a more subtle component of a startle reaction, namely, the orienting reaction (OR). The OR (Sokolov, 1963) or "investigatory reflex" (Pavlov, 1927 ) is observed to occur to stimulus changes in any modality and may be assumed to be a weaker version of the startle, located at one pole of a reflex intensity dimension that includes startle, with defensive reactions at the other more vigorous pole. For these reasons, Stitt, Hoffman, and Marsh (1973) hypothesized that the initial stimulus may be effective because of its OR elicitation, inasmuch as, presumably, the OR component of the startle reflex to S2 would be occluded by a prior occurrence of S1. This occlusion hypothesis implies that inhibition is produced by reflex elicitation and thus it may be inferred that stimulus characteristics, including rise time, which are important for one phenomenon are equally important for the other. Parenthetically it may be noted that the antecedents of the OR are sometimes described as being "sudden" changes in stimulation (for example, Lynn, 1966, p. 11; Pavlov, 1927, p. 44), which suggests a point of similarity in the antecedents of the startle reflex and the OR, although the experimental significance of "sudden" seems not to have been systematically or quantitatively explored in the OR literature. In some contexts, the word is used in the sense of "unexpected, 
without warning," whereas in others it is used as meaning "having an abrupt onset" (as in Pavlov, 1927 , p. 44 , or Sokolov, 1963, p. 105). Only the latter meaning is relevant to the present work on inhibition of the startle reflex in rats, but it is interesting that Landis and Hunt (1939, p. 33-39) point to the significance of each of these variables in the determination of startle behavior in humans.

\section{METHOD}

\section{Part A}

Subjects. The animals were eight naive male albino rats obtained from the Holtzman Co., Madison, Wisconsin, about 15 weeks old at the time of testing. They were run in the light period of a reverse light/dark cycle, and were maintained at all times on ad-lib food and water.

Apparatus. The animals were run in a small cage $(16 \times 7 \times$ $9.5 \mathrm{~cm}$ ) with brass-rod sides and a floor made of two steel plates separated by a $1.2-\mathrm{cm}$ gap. The cage was held firmly in a frame with compression springs. A white-noise background was provided by a Grason-Stadler white-noise generator, the output of which fed a DLK cross-over speaker network. White noise stimuli (S1), also provided by a Grason-Stadler white-noise generator, were passed through a Davan attenuator prior to transmission to the same speaker. Analysis over octave ranges from $125 \mathrm{~Hz}$ to $10 \mathrm{kHz}$ indicated that $\mathrm{S1}$ and the background stimulus had the same power spectrum, which was, however, not rectangular but peaked in the region $1-2 \mathrm{kHz}$ at 8 to $10 \mathrm{~dB}$ higher than that found in the region $6-10 \mathrm{kHz}$. A startle stimulus (S2) provided by a Hewlett-Packard oscillator was delivered after amplification to a Lansing high-frequency tweeter. The two punctate stimuli, S1 and S2, were controlled by electronic switches with adjustable rise times and, after amplification or attenuation, were passed through triac circuits, which were closed only during stimulus delivery. The durations of the stimuli and the interstimulus intervais were controlled by electronic millisecond timers which, like the electronic switches, were built at the University of Rochester. The electronic switches provided a linear increase and decrease in the stimulus intensity over the preset interval. The triac circuits were independently activated and switched in simultaneously with the beginning of the rise and out with the end of the decay. Stimulus intensities were measured on a General Radio sound-level meter (A range) and a General Radio ballistic meter, with a General Radio precision microphone, Type 1560-P7, and are reported with respect to a baseline of $20 \mu \mathrm{N} / \mathrm{m}^{2}$. The shape of the signals was monitored by connecting the output of the sound-level meter to a Tektronix storage CRT, and the output of the electronic switch after amplification but before entry into the speakers was also observed. No transients were ever observed.

An accelerometer mounted on the cage detected the animal's reaction to the acoustic stimuli. The output of the accelerometer was amplified on a Grass polygraph, and was then passed to another channel of the storage CRT. Startle reactions to both S1 and S2 were read as peak-to-peak deflection in millimeters.

The animal cage and stimulus devices were placed in a doublewalled Industrial Acoustic Company sound-attenuated room. Stimulus control, timing gear, and recording apparatus were in a nearby room. The animal was watched over closed-circuit TV. Trials were only given when the animal was quiet and oriented along the major axis of the cage.

Procedure. The animal was placed in the cage in the presence of a steady $65-\mathrm{dB}$ white-noise stimulus for $10 \mathrm{~min}$ prior to the experiment. It was then given two control trials ( $\mathbf{S} 2$ alone), in part for calibration purposes and in part to remove from the experimental data the unusually large responses which sometimes occur on the initial trials. The startle stimulus was a $10-\mathrm{kHz}$ tone, $110 \mathrm{~dB}$ in intensity, with 5-msec rise and decay times, and a 15 -msec period of peak intensity. After the first two control trials, there followed 64 trials arranged in an 8 by 8 Latin square with eight types of trials ordered in eight blocks of trials so that each type occurred just once in the block and at a different place within each block. Each animal had a different square. The eight trial types included six in which $\mathrm{S} 1$ and $\mathrm{S} 2$ were both given, these six being taken from a 2 by 3 factorial design with two levels of stimulus rise/decay time and three levels of stimulus intensity. The two levels of rise/decay time were 5 and $100 \mathrm{msec}$. The total acoustic input in $\mathrm{Sl}$ was held constant by varying the duration of peak intensity: the 5-msec stimulus had a 5 -msec rise time, a 100 -msec period of peak intensity, and a 5-msec decay time; the 100 -msec stimulus had a 100 -msec rise time, a 5 -msec period of peak intensity, and a $100-\mathrm{msec}$ decay time. The three levels of S1 intensity were 70,80 , and $90 \mathrm{~dB}$, each imposed on top of the prevailing $65-\mathrm{dB}$ background stimulus. On paired trials, the delay between S1 and S2 was $145 \mathrm{msec}$, measured from the center of S1 to the onset of S2. The other two of the eight trial types were identical S2-alone control trials, identified for convenience as 1 and 2 in the square but treated jointly in all calculations. The reason for doubling the number of control trials compared to the others was to increase the stability of the control baseline used for all of the calculations. Response amplitudes to S1 and S2 on each trial were averaged for each animal, and the mean amplitudes were converted to relative scores, using for each animal its mean score on the control trials as the $100 \%$ base. Inhibition produced by S1 was quantified by taking the difference between the animal's mean $S 2$ control value and the mean reaction to $\mathbf{S 2}$ on the $\mathrm{S1:S2}$ paired trial, which was then expressed as a percent of the mean $S 2$ value. Reflex elicitation produced by $S 1$ was defined as the mean reaction elicited by $\mathrm{S} 1$ and similarly expressed as a percent of the mean $S 2$ control value.

In the top section of Figure 1, labeled "Part A," may be seen the configuration of the two stimuli differing in rise time and their temporal relationship to the eliciting stimulus, S2. Counting from the midpoint of their rise (at $197 \mathrm{msec}$ ), the midpoint of their peak intensity (at $145 \mathrm{msec}$ ), and the midpoint of their decay (at $92 \mathrm{msec}$ ), the stimuli used as $\mathrm{S1}$ had equal intervals separating them from $S 2$. At four points, however, the stimuli necessarily differed: for the onset of their rise, the $100-\mathrm{msec}$ S1 led the 5-msec $\mathrm{Si}$ by about $47 \mathrm{msec}$, and for the completion of their decay, the 5 -msec $S 1$ led the 100 -msec $S 1$ by the same duration; for the completion of their rise and the onset of their decay, these relationships were reversed. (The exact equating of the two midpoints could not be accomplished with timers adjustable only in milliseconds: the midpoints of the 100 -msec rise time stimulus was

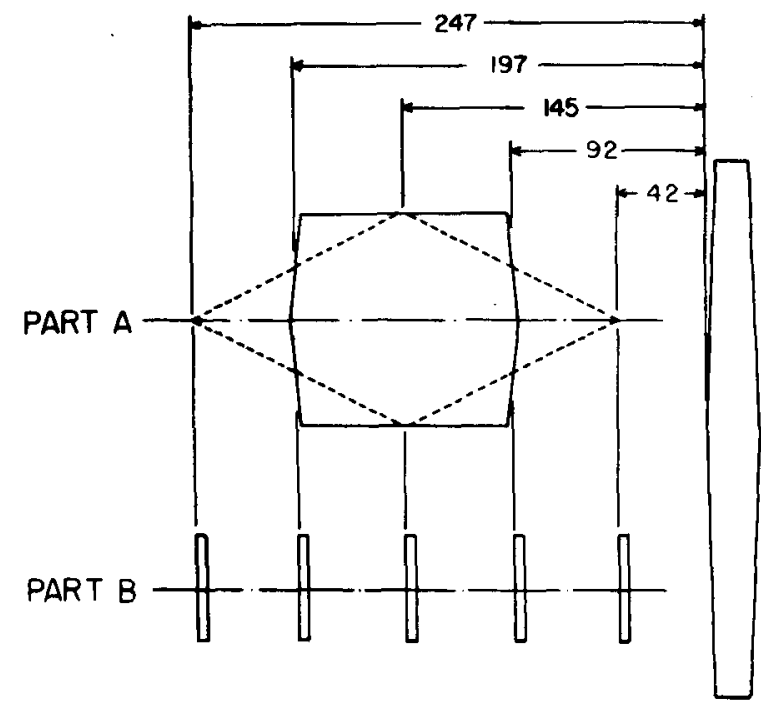

Figure 1. Schematic representation of the stimuli varying in rise/decay time (Part A) and lead time from the eliciting stimulus (Part B), giving the interval in milliseconds between various aspects of $S 1$ and $S 2$. 
145.5 msec rather than 145 msec.) It may be asked whether these temporal differences are of any empirical significance-whether, for example, the 100 -msec stimuli might be in a more favorable temporal position for inhibiting $\mathbf{S} 2$ by virtue of its final offset being but $42 \mathrm{msec}$ away from $\mathrm{S} 2$. This question was asked in a subsidiary experiment, which is Part B.

\section{Part B}

Subjects. The animals were nine naive male albino rats of the same description as those used in Part $\mathrm{A}$.

Apparatus. The same apparatus was used as before.

Procedure. The general procedures, stimulus parameters, and so forth, were the same as those used in Part A. After the first 2 control trials, there followed a block of 60 trials made up from 10 rows of 6 conditions, the order of conditions, the order of conditions within a row being taken from 10 rows of two 6 by 6 Latin square designs. Each row consisted of a control trial of S2 alone and five trials on which S2 was preceded by an S1 with lead times of 247, 197, 147, 92, and $42 \mathrm{msec}$. (Due to an experimental error, $147 \mathrm{msec}$ was used as the midpoint rather than $145 \mathrm{msec}$.) Each $\mathrm{S1}$ was $5 \mathrm{msec}$ in duration and had a 1 -msec rise and decay time. Its intensity was $80 \mathrm{~dB}$, the median intensity used in Part A. The relationship between the two parts is apparent in Figure 1, Part B. Each S1 in Part B is presented at about the time of a change in SI in Part A: at $247 \mathrm{msec}$, one stimulus is at the time of the onset of the $100-\mathrm{msec}$ S1 in Part A; at $197 \mathrm{msec}$, a second stimulus is at the midpoint of the rise of each $S 1$ and approximates, within $3 \mathrm{msec}$, the beginning and end of the rise of the 5-msec S1; at $147 \mathrm{msec}$, a third stimulus is at the (approximate) midpoint of each $\mathrm{Sl}$ and approximates the end of the rise and the beginning of the decay of the $100-\mathrm{msec} \mathrm{S1}$; at $92 \mathrm{msec}$ and at 42 msec, the fourth and fifth stimuli bear analogous relations to the offset of the two stimuli of Part A. The stimuli with separations of 197 and $92 \mathrm{msec}$ from S2 stood in place of both the beginning and the end of the rise and the decay of the 5-msec rise time stimulus, as a 5-msec difference at these separations would make no appreciable difference in the resulting inhibition. Inhibition produced by these stimuli was defined for each subject separately, as before, the difference between the mean reaction to $\mathrm{S} 2$ and the mean reaction to $\mathrm{S} 1$ expressed as a percent of the mean $S 2$ value.

\section{RESULTS AND DISCUSSION}

The outcome of Part A of the experiment is depicted in Figure 2. The differential effectiveness of the rise time variable in controlling reflex elicitation but not reflex inhibition is most apparent. In the lower half of Figure 2, it can be seen that both stimulus rise time and stimulus intensity determined the size of the reflex elicited by S1. Reflex amplitudes increased with increased stimulus intensity $[F(2,14)$ $=13.44, \mathrm{p}<.01]$ and increased with decreased rise time $[\mathrm{F}(1,7)=15.62, \mathrm{p}<.01]$. Rise time became an increasing significant variable as stimulus intensity increased [for their interaction, $F(2,14)=7.51$, $p<.05$ ]. In the upper half of Figure 2 is depicted the strength of reflex inhibition occasioned by these same stimuli. Reflex inhibition was linearly related to the increase in $\mathrm{S} 1$ intensity $[\mathrm{F}(2,14)=31.96, \mathrm{p}<.01]$, but, in contrast to the reflex elicitation data, there was no sign that inhibition was at all affected by differences in stimulus rise times $(F<1)$. The contrast is particularly striking for the two 90 -dB stimuli. The size of the reflex elicited by the stimulus with the 5-msec rise time was about 3 times larger than that produced by the stimulus with the 100 -msec rise time, whereas the amount of inhibition associated with the two stimuli was almost identical $(81.5 \%$ and $79.6 \%$ ).

The outcome of the subsidiary experiment is shown in Figure 3. The relationship between the magnitude of the inhibitory effect and the S1-S2 interval was nonmonotone, as is characteristic of these experiments, with a peak at $92 \mathrm{msec}$. Analysis of variance yielded a significant effect of interstimulus interval $[F(1,8)=5.67, p<.05]$ and a significant cubic trend $[F(4,32)=2.79, p<.05]$. A subsequent critical difference test $(p<.05)$ revealed that average inhibition at the $92-\mathrm{msec}$ separation differed from those obtained at $42 \mathrm{msec}$, on the one side, and at 197 and $247 \mathrm{msec}$, on the other. Although the expectation is that inhibition must continue to decline steadily as the interval increased past $145 \mathrm{msec}$, no other differences were reliable, indicating that in this range of intervals the slope of the function was too slight to be detected by an experiment having the present degree of power.

In earlier experiments, we have found that the interval yielding peak inhibition varies with both qualitative and quantitative aspects of S1 (Ison \& Hammond, 1971), so that peak inhibition with a more intense S1 should occur at an earlier interval,

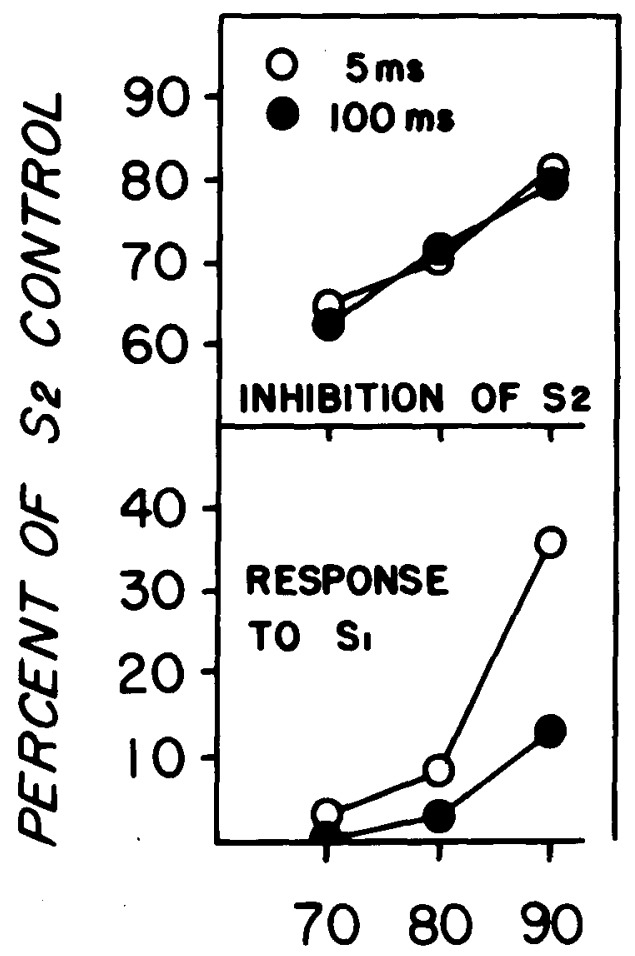

\section{SI INTENSITY (db)}

Figure 2. Startle reflex inhibition (top) and reflex elicitation (bottom) expressed as a percent of the S2-alone control value provided by preliminary stimuli (S1) differing in intensity and rise/decay time (Part A). 


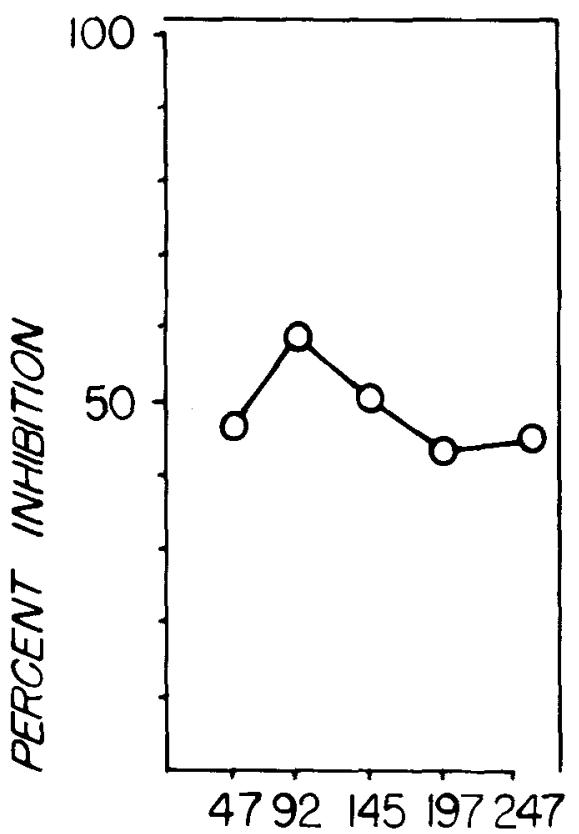

ISI (msec)

Figure 3. Startle reflex inhibition by preliminary stimuli differing in lead time before the eliciting stimulus (Part B).

whereas with a less intense $\mathrm{S} 1$ it should occur at a later interval. It is reasonable to suppose, however, that these present data, collected with an S1 of an intermediate intensity, characterize the central influence which may be expected to have occurred in Part A. The significance of these subsidiary data is that they show that the failure to find a difference between the two inhibitory stimuli used in Part A did not result because the S1 with the gradual onset happened to occupy a more favorable placement with respect to S2, a placement which was sufficient to outweigh the hypothetical inhibitory advantage derived from the other $\mathrm{S} 1$ having a more sudden rise and fall. To the contrary: If one were to define the two stimuli by a concatenation of their onsets and offsets, then it is the sudden stimulus which at offset demonstrably occupied the more favorable position. That no difference attributed to the rise/decay time of the two stimuli was found could mean that it was the gradual stimulus which was the more effective and, thus, overcame an unfavorable position. It is more likely, however, that defining a stimulus as a concatenation of onsets and offsets rather than as a single unitary event is inappropriate. However, it is the case that assigning inhibitory values for the onset and offset of the rise and the onset and offset of the decay and then combining these as four independent events by the rule of Ison et al. (1975) would result in differences which would favor the 5-msec S1 by an amount (about 1\%) too small to be reliably detected.

Under the conditions of this experiment, in Part A it is evident that the reflex-eliciting and reflexinhibiting consequences of an acoustic stimulus do not share identical antecedent conditions, as reflex inhibition was not determined by stimulus rise time. Part of the significance of this finding is that it contradicts those hypotheses concerning the genesis of inhibition which state its central prerequisite to be that the initial stimulus should elicit the startle reflex or some component of that reflex, as, for example, the orienting reflex. In this respect, the data support the finding of two previous experiments in which reflex inhibition and reflex elicitation were manipulated independently. For example, in a threestimulus design, an initial stimulus will inhibit the size of the reflex elicited by the second stimulus without changing the inhibitory effect of that second stimulus on the reflex elicited by the third (Ison \& Krauter, 1974). Further, in the usual two-stimulus design, repetition of the first stimulus will habituate the reflex associated with it without degrading its inhibitory consequence on a second stimulus (Russo, Reiter, \& Ison, 1975). There are now three experiments which reveal that reflex inhibition is invariant with changes in stimulus conditions that have major effects on reflex elicitation, indicating that the mechanisms underlying the two phenomena are different and experimentally separable.

A distinction between the antecedents of reflex elicitation and reflex inhibition may illuminate characteristics of the different physiological processes which underlie them. In her discussion of startle reflex modulation, Graham (1975) pointed to data gathered by Gersuni and his associates (Gersuni, 1965, 1971) which indicate that there are two acoustic systems that can be discriminated on the basis of the temporal characteristics of the stimuli to which they are sensitive. One system is described as having a "short time constant." It responds to stimuli having an abrupt onset, and it has little ability to integrate stimuli over time (e.g., $>10 \mathrm{msec}$ ). Electrophysiological investigations show that groups of cells in this system which fire in unison are responsible for the synchronous evoked potential produced only by stimuli having rapid onsets. In contrast, the second system has a "long time constant." Its response follows the input characteristics of the stimulus and it continues responding to a stable stimulus. Electrophysiological investigations reveal that cells in this system fire asynchronously while the acoustic input continues, and integrate stimulus input over relatively long periods of time.

Graham and Murray (1977) argue that both reflex elicitation and reflex inhibition are produced by the auditory system having the short time constant. The present results suggest that (to the extent that this distinction is applicable to reflex elicitation and inhibition) they are produced by different systems, 
reflex inhibition by that one capable of integrating stimulus input over a long period. Their contrary evidence, suggesting that the inhibitory system does not integrate stimulus input over a long time period, is that, in human subjects, the acoustic startle reaction is inhibited by a punctate $20-\mathrm{msec} \mathrm{S} 1$ as much as it is by a prolonged S1 that overlaps S2 (Graham \& Murray, 1977). This outcome suggested to the authors that S1 onset is of sole importance in determining inhibition. And this may be, but their data are not unequivocal. The rapid rise/decay $(.01 \mathrm{msec})$ of the $1-\mathrm{kHz}$ tone used as $\mathrm{S} 1$ in their experiment yields a broad spectral momentary burst which spreads over the basilar membrane and may actually have made stimulus onset sufficiently louder than the steady state (because of spatial summation) to override differences in temporal summation. (It may be noted that the spread of energy occurring with ultrashort rise times is caused by the way in which the basilar membrane decodes the acoustic input. Although its effect may be the same, it is different in origin from the switching transients which may occur at longer rise times when the control equipment malfunctions. The latter can be controlled by appropriate purchase and/or repair; the former cannot. This limits the range of rise time values that can be properly explored, for even the best equipment cannot prevent the leading edge of a "pure tone" from affecting a considerable portion of the basilar membrane and thus functioning as "noise.") Second, in experiments with rats and with rabbits (Ison \& Hammond, 1971; Ison \& Leonard, 1971), we have found that prolonged stimuli produce reflex facilitation. This may also be true in humans, and a facilitatory consequence of the overlapping S1 may have overridden the increasing inhibitory effect of its longer duration. And finally, and perhaps most important, their prolonged stimulus had no offset and, if we consider onset and offset as two inhibiting events, the short S1 had an advantage derived from its having provided two sources of inhibition.

These speculations are not completely satisfying, because they require that two opposing hypothetical consequences of $\mathrm{Sl}$ balance each other exactly or, at least, exactly in terms of a metric determined by the power of the experiment. A proper investigation of temporal integration of reflex inhibition is needed, and may reveal, in agreement with the conclusions of Graham and Murray (1977), that inhibition is not greater with a longer $\mathrm{S} 1$. If such were the outcome, we must then conclude on an empirical level that neither duration nor abruptness of change of S1 determines its inhibitory effect, which would leave only its subjective intensity and its temporal relation to $\mathrm{S} 2$ as controlling variables. The theoretical significance of this empirical conclusion would be simply that the notion of separate auditory systems having separate time constants is not usefully applied to the understanding of reflex inhibition.

\section{REFERENCES}

Berg, K. M. Elicitation of acoustic startle in the human. Unpublished PhD thesis, University of Wisconsin, 1973.

FLeshler, M. Adequate acoustic stimulus for startle reaction in the rat. Journal of Comparative and Physiological Psychology, $1965,60,200-207$.

Gersuni, G. V. Organization of afferent flow and the process of external signal discrimination. Neuropsychologia, 1965, 3, 95-109.

GERSUNI, G. V. Temporal organization of the auditory function. In G. V. Gersuni (Ed.), Sensory processes at the neuronal and behavioral levels. New York: Academic Press, 1971. Pp. 85-114.

Graham, F. K. The more or less startling effects of weak prestimulation. Psychophysiology, 1975, 12, 238-248.

Graham, F. K., \& Murray, G. M. Discordant effects of weak prestimulation on magnitude and latency of the reflex blink. Physiological Psychology, 1977, 5, 108-114.

Hoffman, H. S., \& Searle, J. L. Acoustic variables in the modification of the startle reaction in the rat. Journal of Comparative and Physiological Psychology, 1965, 60, 53-58.

Ison, J. R., \& HAMMOND, G. R. Modification of the startle reflex in the rat by changes in the auditory and visual environments. Journal of Comparative and Physiological Psychology, 1971, 75, 435-452.

IsON, J. R., \& KRAUTER, E. E. Reflex-inhibiting stimuli and the refractory period of the acoustic startle reflex in the rat. Journal of Comparative and Physiological Psychology, 1974, 86, 420-425.

IsON, J. R., \& LEONARD, D. W. Effects of auditory stimuli on the nictitating membrane reflex of the rabbit (Oryctolagus cuniculus). Joumal of Comparative and Physiological Psychology, 1971, 75, 157-164.

Ison, J. R., Zuckerman, M., \& Russo, J. M. Combination rules for inhibitory stimuli. Journal of Experimental Psychology: Animal Behavior Processes, 1975, 1, 318-325.

KRAUTER, E. E., LeONARD, D. W., \& Ison, J. R. Inhibition of the human eyeblink by a brief acoustic stimulus. Joumal of Comparative and Physiological Psychology, 1973, 84, 246.251.

Landis, C., \& Hunt, W. A. The startle pattern. New York: Farrar \& Rinehart, 1939.

LynN, R. Attention, arousal and the orientation reaction. London: Pergamon Press, 1966.

Pavlov, I. P. Conditioned reflexes. Oxford: Clarendon Press, 1927.

Pinckney, L. A. Inhibition of the startle reflex in the rat by prior tactile stimulation. Animal Learning \& Behavior, 1976, 4, 467-472.

ReIter, L. A., \& IsoN, J. R. Inhibition of the human eyeblink reflex: An evaluation of the Wendt-Yerkes method for threshold detection. Journal of Experimental Psychology: Human Perception and Performance, 1977, 3, 325-336.

Russo, J. M., ReIter, L. A., \& Ison, J. R. Repetitive exposure does not attenuate the sensory impact of the habituated stimulus. Journal of Comparative and Physiological Psychology, $1975,88,665-669$.

Soxolov, Y. N. Perception and the conditioned reflex. London: Pergamon Press, 1963.

Stitt, C. L., Hoffman, H. S., \& Marsh, R. Modification of the rat's startle reaction by termination of antecedent acoustic signals. Journal of Comparative and Physiological Psychology. $1973,84,207-215$.

(Received for publication July 8, 1977 ; revision accepted September 6, 1977.) 